Check for updates

Cite this: RSC Adv., 2017, 7, 53497

\title{
Introduction of inorganic nano-particles into a polymer matrix to restrain the initiation and propagation of electrical trees in the corona condition
}

\begin{abstract}
Chunyu Shang, (D)* Yanqiu Du and Hui Kang
In the corona condition, the initiation and propagation of electrical trees in a polymer matrix originates from the field enhancement effect. Driven by the alternating background electric field in the corona process, an alternating current would pass though the decomposition channel of the electrical tree, stimulating an enhanced local electric field on the tip of the electrical tree. When inorganic nano-particles with high corona-resistibility were introduced into the polymer matrix, in the corona process, the inorganic nanoparticles were aggregatively deposited in the decomposition channels of the electrical trees and on the surface of the composite material. The decomposition channels were blocked and the alternating current was shut off, eliminating the enhanced local electric field on the tip of the electrical tree. As a result, the initiation and propagation of electrical trees were restrained and improved coronaresistibility was achieved for the polymer/nano-particles composite material.
\end{abstract}

Received 24th August 2017

Accepted 12th November 2017

DOI: 10.1039/c7ra09382a

rsc.li/rsc-advances

\section{Experimental section}

Polyimide (PI) film and $\mathrm{PI} / \mathrm{TiO}_{2}$ composite films $\left(\mathrm{TiO}_{2}\right.$ content $=$ $0 \mathrm{wt} \%, 2 \mathrm{wt} \%, 4 \mathrm{wt} \%, 6 \mathrm{wt} \%, 8 \mathrm{wt} \%, 10 \mathrm{wt} \%, 12 \mathrm{wt} \%, 14 \mathrm{wt} \%$, and $16 \mathrm{wt} \%)$ were synthesized by in situ polymerization. In an ultrasonic bath, $N, N$-dimethylacetamide (DMAC, supplied by Tianjin Fuyu Fine Chemical Co., Ltd.) and $\mathrm{TiO}_{2}$ nano-particles with sizes of 30-100 $\mathrm{nm}$ (supplied by Beijing NaChen Technology Co., Ltd.) were added into a three-necked flask, and a stable suspension was formed after sufficient stirring and ultrasonic oscillation. A certain amount of 4,4'-oxy dianiline (ODA, supplied by Sinopharm Chemical Reagent Co., Ltd.) and pyromellitic dianhydride (PMDA, supplied by Sinopharm Chemical Reagent Co., Ltd.) were added into the suspension; after stirring, the mixture was cast on a glass substrate and dried at $80^{\circ} \mathrm{C}$ for $6 \mathrm{~h}$. After being successively cured at $120^{\circ} \mathrm{C}, 200^{\circ} \mathrm{C}$ and $300{ }^{\circ} \mathrm{C}$ for $2 \mathrm{~h}$, PI film and $\mathrm{PI} / \mathrm{TiO}_{2}$ composite films with thickness of about $30 \mu \mathrm{m}$ were obtained. The XRD characterizations of $\mathrm{PI}, \mathrm{TiO}_{2}$ and $\mathrm{PI} / \mathrm{TiO}_{2}$ were undertaken with a Rigaku$\mathrm{D} / \mathrm{max} 2500 \mathrm{X}$-ray diffractometer using $\mathrm{Cu}$ Ka radiation. The time-to-breakdown measurements were performed under an alternating voltage of $1.7 \mathrm{kV}$ in a rod-plate electrode system (IEC60343). An Al electrode was evaporated on each of the PI/ $\mathrm{TiO}_{2}$ composite films (including the PI film). In the measurements, a tiny air gap was retained between the film and the rod electrode. The morphologies of the $\mathrm{PI} / \mathrm{TiO}_{2}$ composite films were characterized using an environmental scanning electron microscope (ESEM) type Quanta 200 operated at $20 \mathrm{kV}$ with secondary and backscattering electrons in high-vacuum mode. 


\section{Results and discussion}

\subsection{Initiation and propagation of electrical trees in} a polymer matrix owing to the field enhancement effect

In our research work, we have revealed that in theory the initiation and propagation of electrical trees in polymer matrix originate from the field enhancement effect. ${ }^{8}$ Herein, in order to carry out the discussions in this paper, the initiation and propagation mechanisms of electrical trees need to be referred to.

When a conductor of a certain aspect ratio $R_{l 0 / \rho 0}$ is parallel to the background electric field $E_{\text {background }}$ between a pair of electrodes and the conductor is connected to one of the electrodes, free charges would be present on the top surface of the conductor. The radial and axial components of the electric field $E(\rho, l)$ around the top of the conductor may be given as:

$$
\begin{aligned}
& E_{\rho}(\rho, l)=E_{\text {background }} \times\left[k l_{0} \times \frac{N_{1}(k \rho)}{N_{0}\left(k \rho_{0}\right)} \times \frac{\mathrm{e}^{k l}-\mathrm{e}^{k(2 d-l)}}{\mathrm{e}^{k l_{0}}-\mathrm{e}^{k\left(2 d-l_{0}\right)}}\right] \\
& E_{l}(\rho, l)=E_{\text {background }} \times\left[k l_{0} \times \frac{N_{0}(k \rho)}{N_{0}\left(k \rho_{0}\right)} \times \frac{\mathrm{e}^{k l}+\mathrm{e}^{k(2 d-l)}}{\mathrm{e}^{k l_{0}}-\mathrm{e}^{k\left(2 d-l_{0}\right)}}-1\right]
\end{aligned}
$$

where $N_{0}$ and $N_{1}$ are the zero-order and first-order Neumann functions, respectively; $\rho$ is a radial coordinate and $l$ is an axial coordinate; $d$ is the distance between the electrodes; and $k$ is a positive constant. ${ }^{9-11}$ Based on formulas (1a) and (1b), it can be determined that when the length $l_{0}$ is larger or the radius $\rho_{0}$ is smaller, the local electric field $E_{\text {local }}$ around the top of conductor $\left(\rho \rightarrow \rho_{0}, l \rightarrow l_{0}\right.$ ) should be stronger. When $R_{l 0 / \rho 0}$ is large, the field enhancement effect is significant and $E_{\text {local }}$ may be much stronger than $E_{\text {background }}$.

In the corona condition, a polymer matrix is under the action of a strong alternating background electric field $E_{\text {background }}(t)$. The electrode-polymer interface is far from ideal at the microscopic scale. In a certain spot, there may be a sharp protrusion erected on the electrode surface (a conductive impurity particle or a defect of higher conductivity, etc.), embedded in the polymer matrix. ${ }^{1,2}$ In such a spot, stimulated by the alternating background electric field $E_{\text {background }}(t)$ in the corona process, a field enhancement effect would be present around the projection top, as indicated in Fig. 1.

When $E_{\text {background }}(t)$ is negative (see Fig. 1), free charges $\left(Q_{\text {free }}(t)<0\right)$ would be distributed on the interface between the protrusion top and the polymer matrix (top-matrix interface), trapped in interface states with different energies $\left(E_{\mathrm{it}}\right)$ in the energy gap of the polymer matrix; the local electric field $E_{\text {local }}$ is oriented toward the protrusion top. Owing to the high strength of $E_{\text {local }}(t)$, the distributions of electrons in the interface states may not be stable; a trapped electron may be emitted into the polymer matrix via the quantum tunneling effect. ${ }^{12,13}$ Leaving the top-matrix interface, an electron should be accelerated by $E_{\text {local }}(t)$. After being constantly scattered, the electron should be finally trapped in a localized state, becoming a space charge in the polymer matrix around the protrusion top, as indicated in Fig. 1. When $E_{\text {background }}(t)$ is positive, free charges $\left(Q_{\text {free }}(t)>0\right)$

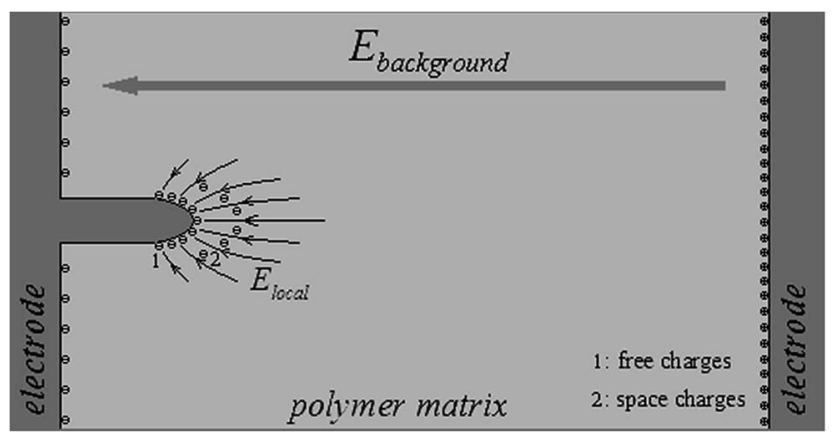

Fig. 1 Schematic distributions of local electric field, free charges and space charges around the projection top when the background electric field is negative.

would be distributed on the top-matrix interface; the local electric field $E_{\text {local }}$ is oriented outward from the protrusion top. Owing to the high strength of $E_{\text {local }}(t)$, the distributions of space charges (electrons) in the localized states in the polymer matrix around the protrusion top may not be stable; a trapped electron in the localized state may be emitted via the quantum tunneling effect. After being emitted, an electron should be accelerated by $E_{\text {local }}(t)$, be constantly scattered, and approach the protrusion top until it is recovered onto the top-matrix interface. In the corona condition, the magnitude of the alternating background electric field $E_{\text {background }}(t)$ may be rather strong, bringing about a much stronger local electric field $E_{\text {local }}(t)$ around the protrusion top. Owing to the high strength of $E_{\text {local }}(t)$, the accumulated kinetic energies $\left(E_{\mathrm{k}}\right)$ of electrons in the accelerating processes may be high enough to break the chemical bonds, causing the decomposition of polymer material around the protrusion top, initiating an electrical tree on the surface of the polymer matrix. ${ }^{14}$

In the propagation of an electrical tree in a polymer matrix, different low-molecular decomposition products (especially carbonization products) would be deposited on the side wall of the decomposition channel, presenting a certain conductivity for the electrical tree. As a result, the electrical tree may be seen to be a quasi-conductor in the corona process. ${ }^{\mathbf{8 1 5}-18}$ Driven by the alternating background electric field $E_{\text {background }}(t)$, the alternating current $I_{\text {channel }}(t)$ would pass though the decomposition channel of the electrical tree, an alternating electric quantity $Q_{\text {space }}(t)$ of space charges would be present on the tip of the electrical tree and an enhanced local electric field $E_{\text {local }}(t)$ would be present around the tip of the electrical tree. Based on experiment, it can be determined that the diameter of an electrical tree is of micrometer magnitude, and the tip of an electrical tree is even finer in size. Consequently, the magnitude of $Q_{\text {space }}(t)$ on the tip of the electrical tree should be rather small. For a sinusoidal alternating electric field $E_{\text {background }}(t)$, in the stable case, the alternating electric quantity $Q_{\text {space }}(t)$ on the tip of the electrical tree and the alternating current $I_{\text {channel }}(t)$ passing though the decomposition channel of the electrical tree may be given as:

$Q_{\text {space }}(t)=Q_{\max } \times \sin \omega t$ 


$$
I_{\text {channel }}(t)=\omega \times Q_{\max } \times \cos \omega t
$$

where $Q_{\max }$ is the magnitude of $Q_{\text {space }}(t)$ and $\omega$ is the angular frequency of $E_{\text {background }}(t)$. Since $Q_{\max }$ is rather small, the alternating current $I_{\text {channel }}(t)$ should be rather weak when $\omega$ is not very high. The acceleration of electrons in the enhanced local electric field $E_{\text {local }}(t)$ around the tip of the electrical tree results in the decomposition of the polymer material, driving the propagation of an electrical tree in the polymer matrix.

\subsection{Introduction of inorganic nano-particles into a polymer matrix to restrain the initiation and propagation of electrical trees in the corona condition}

In view of the initiation and propagation mechanisms of electrical trees in a polymer matrix, inorganic nano-particles with high corona-resistibility may be deliberately introduced into the polymer matrix and improved corona-resistibility may be expected for the polymer/nano-particles composite material.

In our research, taking polyimide (PI, widely applied in engineering insulation) as a typical polymer material and titanium dioxide nano-particles $\left(\mathrm{TiO}_{2}\right)$ as a typical inorganic component with high corona-resistibility, a series of experiments have been carried out. A PI/TiO ${ }_{2}$ composite material was formed by in situ polymerization. X-ray diffraction measurements were performed for $\mathrm{PI}, \mathrm{TiO}_{2}$ and $\mathrm{PI} / \mathrm{TiO}_{2}$ composite material, and the corresponding XRD data are presented in Fig. 2. The characteristic diffraction peaks were assigned to the $\mathrm{TiO}_{2}$ rutile phase and the broad band was assigned to the amorphous phase of the PI polymer matrix. With the introduction of $\mathrm{TiO}_{2}$ nano-particles into the PI polymer matrix, the characteristic diffraction peaks of $\mathrm{TiO}_{2}$ were superimposed on the broad band of PI in the XRD data of the $\mathrm{PI} / \mathrm{TiO}_{2}$ composite material. To achieve comparability in the corona-resistibility measurements, the corona conditions were set to be uniform in all the experiments. With an increase in $\mathrm{TiO}_{2}$ content in the $\mathrm{PI} / \mathrm{TiO}_{2}$ composite material, an improvement in coronaresistibility (indicated by time-to-breakdown) was apparent when the $\mathrm{TiO}_{2}$ content was not very high. The time-tobreakdown of $\mathrm{PI} / \mathrm{TiO}_{2}$ composite film with $\mathrm{TiO}_{2}$ content of

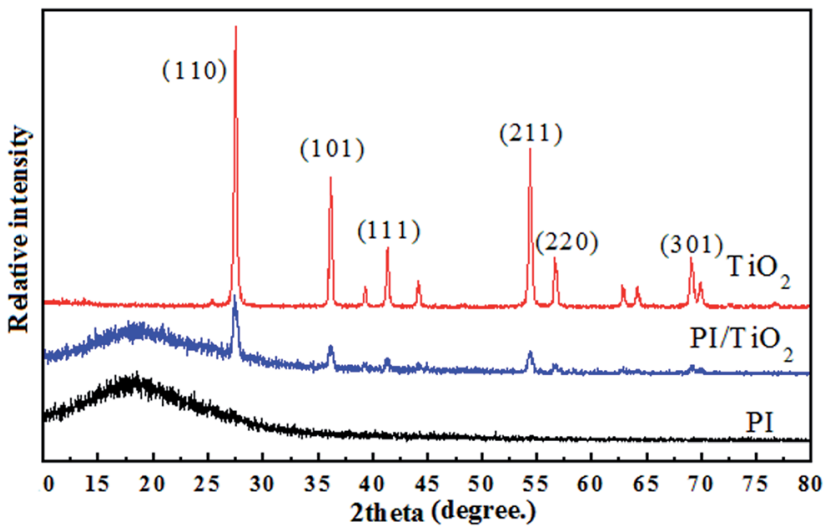

Fig. 2 X-ray diffraction data for $\mathrm{PI}, \mathrm{TiO}_{2}$ and $\mathrm{Pl} / \mathrm{TiO}_{2}$ composite material.

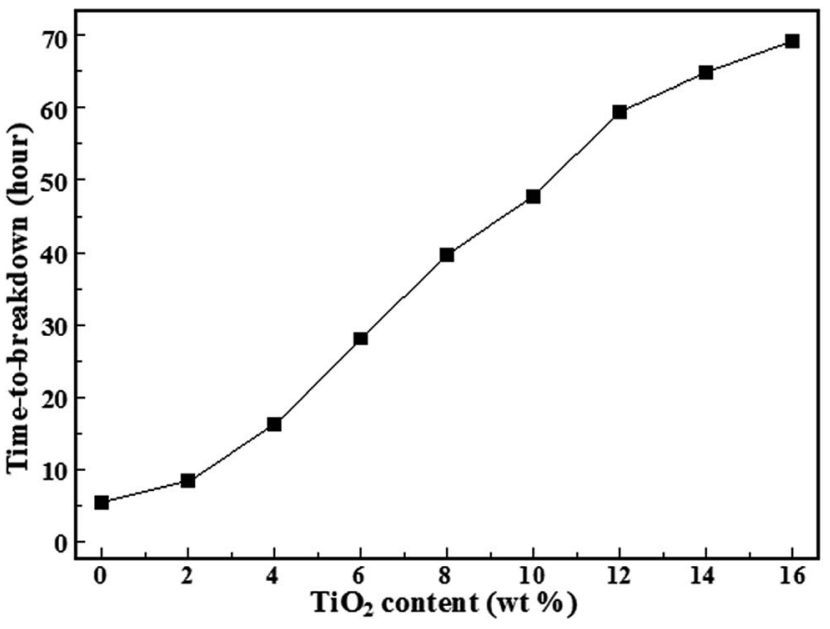

Fig. 3 Time-to-breakdown versus $\mathrm{TiO}_{2}$ content relations for $\mathrm{PI} / \mathrm{TiO}{ }_{2}$ composite films.

0 wt\%, 2 wt\%, 4 wt\%, 6 wt $\%, 8$ wt $\%, 10$ wt $\%, 12$ wt $\%, 14$ wt\%, and $16 \mathrm{wt} \%$ was $5.5 \mathrm{~h}, 8.4 \mathrm{~h}, 16.5 \mathrm{~h}, 28.7 \mathrm{~h}, 39.9 \mathrm{~h}, 47.8 \mathrm{~h}, 59.4 \mathrm{~h}$, $64.9 \mathrm{~h}$ and $68.8 \mathrm{~h}$, respectively. The gradual increase in time-tobreakdown of the composite material indicated the improvement in corona-resistibility achieved by introducing inorganic nano-particles into the polymer matrix, as presented in Fig. 3.

\subsection{Mechanisms in the improved corona-resistibility for the polymer/nano-particles composite material}

In the propagation of an electrical tree in a polymer matrix, driven by the alternating background electric field $E_{\text {background }}(t)$ in the corona condition, the alternating current $I_{\text {channel }}(t)$ would pass though the decomposition channel of the electrical tree to present the alternating electric quantity $Q_{\text {space }}(t)$, stimulating the field enhancement effect on the tip of the electrical tree. If the decomposition channel of the electrical tree, as a quasiconductor, were to be blocked by a certain means, $I_{\text {channel }}(t)$ may be shut off and $Q_{\text {space }}(t)$ may be eliminated, dissipating the local electric field $E_{\text {local }}(t)$. As a result, the decomposition of the polymer material, i.e., the propagation of the electrical tree, may be restrained. Similarly, the initiation of an electrical tree on the surface of polymer matrix may be restrained as well.

In the corona condition, the corona-resistibility is not uniform in a polymer/nano-particles composite material, so the propagation of electrical trees would inevitably bypass the inorganic nano-particles owing to the high corona-resistibility they possess. In this process, the polymer material around the inorganic nano-particles would gradually decompose, forming low-molecular decomposition products, and evaporating. Consequently, the inorganic nano-particles would be left in the decomposition channels of the electrical trees and on the surface of the composite material. Owing to the alternating current $I_{\text {channel }}(t)$ passing though the decomposition channels of the electrical trees (quasi-conductors in the corona process), a thermal effect would be present in the decomposition channels. Meanwhile, the surface energy of the inorganic nanoparticles is much higher than that of the corresponding bulk 


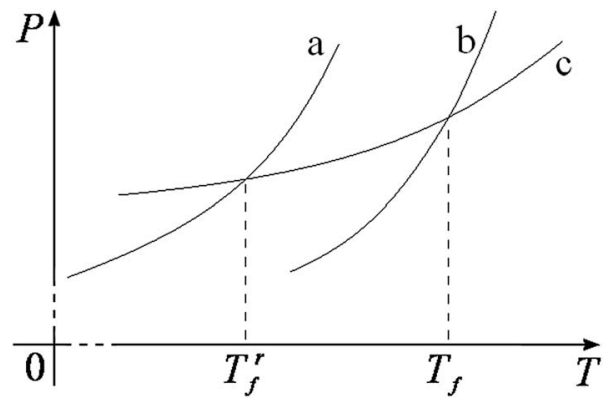

Fig. 4 Schematic relations between vapor pressure and temperature. (a) Microscopic crystal; (b) macroscopic crystal; (c) liquid phase.

material. With the propagation of electrical trees, the inorganic nano-particles left in the decomposition channels and on the surface of the composite material would be aggregatively deposited owing to the high surface energy and the thermal effect in the corona condition. The decomposition channels of the electrical trees would be blocked by the deposited inorganic nano-particles, shutting off the alternating current $I_{\text {channel }}(t)$ in the corona condition. Consequently, the field enhancement effect would be eliminated on the tip of the electrical tree and the propagation of electrical trees in the composite matrix may be restrained. With the decomposition of polymer material, the surface layer of the composite matrix would gradually collapse, with the deposition of inorganic nano-particles on the surface of the composite matrix. Consequently, the initiation of electrical trees on the surface of the composite matrix may be restrained as well.

In addition to the high surface energy of inorganic nanoparticles, the melting point decreasing effect is another specific reason causing the deposited inorganic nano-particles to be aggregately deposited in the corona process. According to thermodynamics, the melting point of a certain crystal material is the specific temperature at which the solid and liquid phases possess the same vapor pressure. ${ }^{19}$ With an increase in temperature, the vapor pressures of solid and liquid phases would all increase, but the vapor pressure of the liquid phase would increase more slowly. ${ }^{19}$ Meanwhile, the vapor pressure of microscopic crystal particles would be higher than that of the macroscopic crystal. ${ }^{\mathbf{1 9 2 0}}$ The relations between vapor pressure and temperature are schematically presented in Fig. 4. It is apparent that the melting point of microscopic crystal particles would be lower than that of the homogeneous macroscopic crystal. In the literature, the following relation is given,

$$
\Delta T=T_{\mathrm{f}}-T_{\mathrm{f}}{ }^{r}=\frac{2 V_{\mathrm{m}} \times \sigma \times T_{\mathrm{f}}}{\Delta_{\mathrm{s}}^{1} H_{\mathrm{m}} \times r}
$$

where $T_{\mathrm{f}}$ and $T_{\mathrm{f}}^{r}$ are the melting points of the macroscopic crystal and microscopic crystal particles, respectively; $V_{\mathrm{m}}$ is the molar volume; $\sigma$ is the surface tension; $\Delta_{\mathrm{s}}^{1} H_{\mathrm{m}}$ is the melting heat; and $r$ is the size of crystal particles. ${ }^{21}$ In view of formula (3),
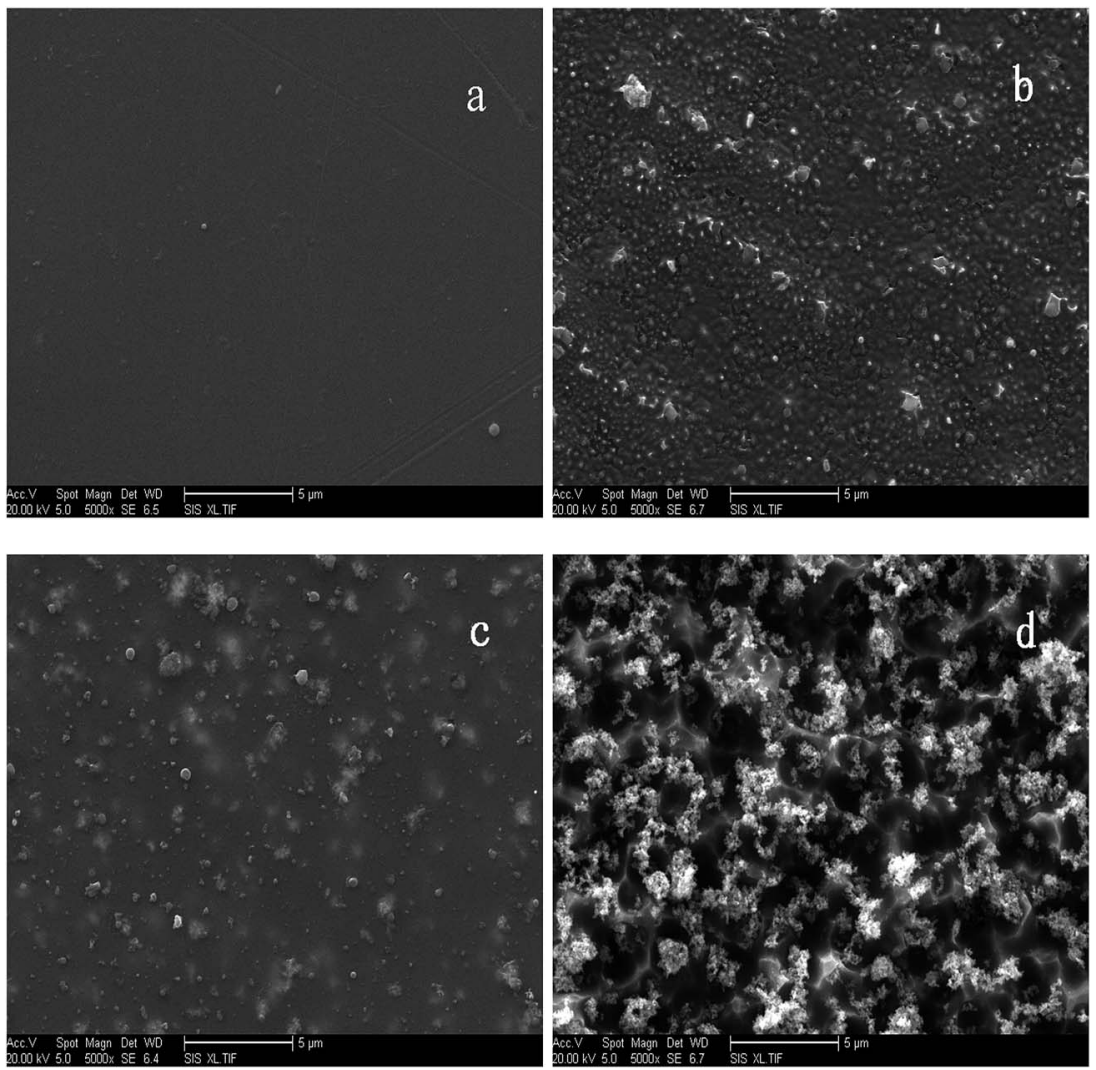

Fig. $5 \mathrm{SEM}$ morphologies of pre-corona PI film (a), post-corona PI film (b), pre-corona $\mathrm{PI} / \mathrm{TiO}_{2}$ composite film (c) and post-corona $\mathrm{PI} / \mathrm{TiO} 2$ composite film (d). 
it may be expected that the melting point decreasing effect is significant when the crystal size drops to the nano-scale. Owing to the melting point decreasing effect, the deposited inorganic nano-particles would be more aggregatively deposited in the decomposition channels of electrical trees and on the surface of the composite material in the corona process. As a result, the initiation and propagation of electrical trees would be further restrained.

Since the initiation and propagation of electrical trees in the corona condition may be restrained by introducing inorganic nano-particles into the polymer matrix, improved coronaresistibility for a polymer/nano-particles composite material would inevitably be achieved. The SEM morphologies of the PI film and $\mathrm{PI} / \mathrm{TiO}_{2}$ composite film before and after the corona experiments are presented in Fig. 5.

As verified by experiment and reported in the literature, inorganic nano-particles are more suitable than homogeneous inorganic micron-particles for introduction into the polymer matrix to restrain the initiation and propagation of electrical trees in the corona condition. Based on the initiation and propagation mechanisms of electrical trees presented in our research, the corresponding reasons may be exactly clarified. In the polymer/micron-particles composite material, with the decomposition of polymer material propagating into the composite matrix in the corona process, inorganic micronparticles would be deposited in the decomposition channel and on the surface of the composite matrix. It should be noted that the interspaces between the deposited micron-particles should be of sub-micrometer magnitude, which are much larger than those in the deposited nano-particles. On the other hand, in typical polymer materials, the diameter size of an electrical tree ranges from a few tenths of a micrometer to tens of micrometers. Owing to the relatively large interspaces between the deposited micron-particles, the decomposition channels of electrical trees may not be sufficiently blocked and the alternating current $I_{\text {channel }}(t)$ in the decomposition channel may not be sufficiently shut off in the corona process. As a result, the propagation of electrical trees may not be effectively restrained. Similarly, the initiation of electrical trees may not be effectively restrained as well in the corona process. Consequently, the improvement in corona-resistibility may not be sufficiently achieved by introducing inorganic micron-particles into the polymer matrix.

\section{Conclusions}

As a long-term electrical erosion process, electrical trees are initiated and propagated in a polymer matrix in the corona condition. The initiation and propagation of electrical trees originate from the field enhancement effect. In view of the initiation and propagation mechanisms of electrical trees, inorganic nano-particles with high corona-resistibility may be introduced into the polymer matrix and a high coronaresistibility may be expected for a polymer/nano-particles composite material. In experiments, an apparent improvement in corona-resistibility was achieved for a $\mathrm{PI} / \mathrm{TiO}_{2}$ composite material. The time-to-breakdown of $\mathrm{PI} / \mathrm{TiO}_{2}$ composite film with a $\mathrm{TiO}_{2}$ content of $0 \mathrm{wt} \%, 2 \mathrm{wt} \%, 4 \mathrm{wt} \%$, $6 \mathrm{wt} \%, 8 \mathrm{wt} \%, 10 \mathrm{wt} \%, 12 \mathrm{wt} \%, 14 \mathrm{wt} \%$, and $16 \mathrm{wt} \%$ was $5.5 \mathrm{~h}$, 8.4 h, 16.5 h, 28.7 h, 39.9 h, 47.8 h, 59.4 h, 64.9 h and 68.8 h, respectively. In addition, in view of the initiation and propagation mechanisms of electrical trees, inorganic nano-particles are more suitable than homogeneous inorganic micronparticles for introduction into the polymer matrix owing to the relatively large gaps between the deposited micronparticles, as verified by experiment.

\section{Conflicts of interest}

There are no conflicts to declare.

\section{Acknowledgements}

This work was financially supported by Heilongjiang postdoctoral scientific research developmental fund (LBH-Q15124), Science and Technological Research Project of Heilongjiang Provincial Education Department (1253052).

\section{References}

1 R. Gautam, R. Vinu, R. Sarathi, S. Acharya, M. Kumar and A. Sharma, IEEE Trans. Dielectr. Electr. Insul., 2016, 23, 1652.

2 Y. Tanaka, G. Chen, Y. Zhao, A. E. Davies, A. S. Vaughan and T. Takada, IEEE Trans. Dielectr. Electr. Insul., 2003, 10, 148.

3 H. Kim, S. Nam and J. Park, Semicond. Sci. Technol., 2010, 25, 105006.

4 R. Sarathi, A. Nandini and M. G. Danikas, JEE, J. Electron. Eng., 2011, 62, 73.

5 X. Shang, D. Han, M. Liu and G. Zhang, RSC Adv., 2017, 7, 5055.

6 L. R. Zhou, G. N. Wu and B. Gao, IEEE Trans. Dielectr. Electr. Insul., 2009, 16, 1143.

7 J. W. Zha, Z. M. Dang and H. T. Song, J. Appl. Phys., 2010, 108, 09113.

8 C. Y. Shang, J. X. Zhao, X. Q. Wang, H. Y. Xia and H. Kang, $R S C A d v ., 2015$, 5, 16993.

9 L. Q. Guo, D. Han, H. Ma, K. Y. Song, H. N. Wu and S. Wang, J. Synth. Cryst., 2007, 36, 847.

10 C. Y. Shang, J. X. Zhao and X. Q. Wang, Phys. Chem. Chem. Phys., 2016, 18, 3482.

11 S. H. Guo, Electrodynamics, Higher Education Press, 1997. 12 J. Y. Zeng, Quantum mechanics, Science Press, Beijing, 2008. 13 E. K. Liu, B. S. Zhu and J. S. Luo, Semiconductor Physics, National Defense Industry Press, 2015.

14 X. Shang, Y. Li, Q. Zhan and G. Zhang, New J. Chem., 2016, 40, 1111.

15 S. Misak, J. Fulnecek, T. Jezowicz, T. Vantuch and T. Burianek, Power Engineering and Electrical Engineering, 2017, vol. 1, p. 21.

16 X. R. Chen, Y. Xu, M. Wang, W. H. Yang, Y. Liu and X. L. Cao, High Voltage Engineer, 2012, vol. 38, p. 645.

17 X. R. Chen, Y. Xu, Y. Liu and X. L. Cao, Acta Phys. Sin., 2012, 61, 087701. 
18 G. N. Wu, J. D. Wu, K. Zhou, B. Gao, X. X. Guo and W. G. Wang, Chinese Society for Electrical Engineering, 2009, 29, 13.

19 B. H. Jiang, Material Thermodynamics, Shanghai Jiaotong University Press, Shanghai, 1999.
20 B. X. Du, Y. Q. Xing, J. X. Jin, P. H. Huang and M. Xiao, IEEE Trans. Appl. Supercond., 2016, 26, 1051.

21 M. J. Maclachlan, L. Manners and G. A. Ozin, Adv. Mater., 2000, 12, 675 . 